\title{
Network Economics and the Environment: Insights and Perspectives
}

\author{
Sergio Currarini ${ }^{1}$ - Carmen Marchiori $^{2}$. \\ Alessandro Tavoni ${ }^{3}$
}

Accepted: 11 June 2015

(C) Springer Science+Business Media Dordrecht 2015

\begin{abstract}
Local interactions and network structures appear to be a prominent feature of many environmental problems. This paper discusses a wide range of issues and potential areas of application, including the role of relational networks in the pattern of adoption of green technologies, common pool resource problems characterized by a multiplicity of sources, the role of social networks in multi-level environmental governance, infrastructural networks in the access to and use of natural resources such as oil and natural gas, the use of networks to describe the internal structure of inter-country relations in international agreements, and the formation of bilateral "links" in the process of building up an environmental coalition. For each of these areas, we examine why and how network economics would be an effective conceptual and analytical tool, and discuss the main insights that we can foresee.
\end{abstract}

Keywords Networks - Environmental externalities - Technological diffusion - Gas pipelines $\cdot$ Common-pool resources $\cdot$ Multi-level governance $\cdot$ Coalitions

Sergio Currarini

s.currarini@unive.it; sc526@le.ac.uk

Carmen Marchiori

c.marchiori@1se.ac.uk

Alessandro Tavoni

a.tavoni@1se.ac.uk

1 Department of Economics, University of Leicester and Universita' Ca' Foscari di Venezia, Leicester, UK

2 Department of Geography and Environment, Grantham Research Institute on Climate Change and the Environment, London School of Economics and Political Science, Houghton Street, London WC2A 2AE, UK

3 Grantham Research Institute on Climate Change and the Environment, London School of Economics and Political Science, London, UK 


\section{Introduction}

Recent research has shown how explicitly modelling the network structure of social and economic relations can provide significant theoretical insights, as well as account for previously unexplained empirical observations. Relevant areas of application range from labour markets (Calvo-Armengol 2004; Calvo-Armengol and Jackson 2004), to the diffusion of opinions and diseases (Jackson 2008), trade and financial markets (Eliott et al. 2013), R\&D collaborations (Goyal and Moraga-Gonzalez 2001), friendship and peer effects identification (Currarini et al. 2009, 2010), and the adoption of health related behaviours (Christakis and Fowler 2002).

Network theory is particularly well-suited to analysing problems where social distance affects the nature and extent of economic interactions. In a network, agents interact only with a subset of other agents called the neighbours. For instance, in labour markets, information on job vacancies mainly flows along social ties. Likewise, people's behaviours and habits are affected by those of friends, relatives and colleagues with whom they interact, whom they imitate, and whose actions have an impact on their welfare. Research in network economics has addressed two distinct, though strictly related, issues: (i) how network structures affect the behaviour of social and economic actors; (ii) what incentives agents face in forming the network by means of link creation and deletion (which in turn begs the question how these incentives relate to social incentives, and how efficient are the resulting architectures). Investigation of the above issues has shown that the network structure of career advice can generate unemployment patterns that match the observed correlation and persistence of unemployment much better than classical models do (e.g., Calvo-Armengol and Jackson 2004; Calvo-Armengol 2004). Furthermore, we have learned that the effect of changing the topology of a social network crucially depends on the strategic features of social interactions (i.e. whether they are substitutes or complements) (e.g., Galeotti et al. 2010); and that the ethnic biases in the way students form friendships originate both from institutional constraints and from preferences that are not race-blind but favour one's own ethnic group (e.g., Currarini et al. (2009)).

Local interactions and network structures appear to be a prominent feature of many environmental problems. Without having the ambition to be exhaustive, this paper nonetheless considers a wide range of issues and potential areas of application, including: (i) the role of relational networks in the pattern of adoption and the speed of diffusion of green technologies; (ii) common pool resource problems characterized by a multiplicity of sources and users interlinked by an extraction network; (iii) the role of social networks in multi-level environmental governance; (iv) infrastructural networks in the access to and use of natural resources such as oil and natural gas; (v) the use of networks to describe the internal structure of inter-country relations in international agreements, and how this affects the stability of cooperation; and (vi) the formation of bilateral "links" in the process of building up an environmental coalition.

For each of these areas, we examine why and how network economics would be an effective conceptual and analytical tool, and discuss the main insights that we can foresee. We do this by reviewing relevant yet still limited contributions within this emerging research field, discussing new frameworks of analysis, and identifying open issues and questions for future research.

The paper is organised as follows. Section 2 introduces the fundamental elements of network analysis, and presents some of the key indices that are used to capture the structural features of a network and compare different architectures. Furthermore, it discusses how the 
network is likely to affect agents' actions, behaviours and welfare; and what forces/incentives are behind the process of network formation. In Sect. 3 we seek to map the linkages between network economics and the environment by focusing on specific environmental issues/areas and analysing in greater detail how the use of networks can provide new insights for both theory and practice. Section 4 draws some conclusions.

\section{Network Economics: Key Features and Concepts}

In this section we introduce some fundamental concepts and notations about networks. For a more complete and detailed treatment we refer the reader to Jackson (2008).

\subsection{Definitions}

\subsubsection{Networks}

We define a network starting from a set $N$ of nodes. In applications, nodes usually represent socio economic agents, such as firms, consumers, countries, etc ... A network $g$ can be defined as a subset of the set of all pairs of elements in $N: g \subseteq\{i j: i \in N, j \in N\}$. When the order of pairs matters, we say the network is directed, otherwise we say that the network is undirected (that is, in an undirected network $i j \in g \rightarrow j i \in g$ ). A pair $i j \in g$ is called a link or a tie. Although links can carry an associated real number, which is usually interpreted as the strength of the link, in most economic applications such weights are set to either 1 (the link is there) or 0 (the links is absent), and $g$ is a non-weighted network. We will denote by $g-i j$ the network obtained by deleting the link $i j$ from $g$, and by $g+i j$ the network obtained by adding the link $i j$ to $g$.

An alternative way to represent a network is by means of the adjacency matrix $G$, whose generic entry $g_{i j}$ measures the strength of the link between nodes $i$ and $j$ in $g$. When the network is undirected, the adjacency matrix is symmetric; when links are not weighted, the matrix only contains zeros and ones. The generic element $g_{i j}^{m}$ of the $m-t h$ power of the matrix $G$ counts how many paths of length $m$ are present in $g$ between $i$ and $j$.

The neighbourhood of node $i$ in the non-weighted network $g$ is the set of nodes that are linked to $i$ in $g$. The number of such nodes-called the neighbours of $i$-is called the degree of $i$ in $g$. If all nodes are linked to all other nodes we have the complete network. Notable architectures include (i) minimally connected networks (trees); (ii) regular networks, where all nodes have the same degree (a special case is the circle, where all nodes have two neighbours); and (iii) core-periphery architectures, which are networks where a subset of nodes - the core-is linked to all nodes in the network and the rest of the nodes are only linked to nodes in the core (a special case is the star, where the core includes a single node). See Fig. 1.

\subsubsection{Paths and Connectedness}

A walk in the network $g$ is a sequence of adjacent links in $g$. Formally, a walk is a sequence $\left\{i_{0} i_{1}, i_{1} i_{2}, \ldots, i_{m-1} i_{m}\right\}$ such that $i_{p-1} i_{p} \in g$ for all $p=1, \ldots, m$. When such walk exists, we say that the two nodes $i_{0}, i_{m}$ are connected in $g$. A walk such that $i_{0}=i_{m}$ is called a cycle. When the walk never goes twice through the same node we have a path. When there are several paths connecting nodes $i_{0}$ and $i_{m}$, we consider the shortest of these paths to define 


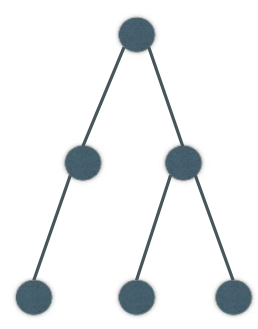

Tree

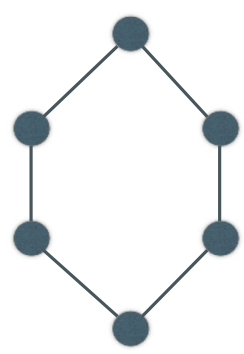

Circle

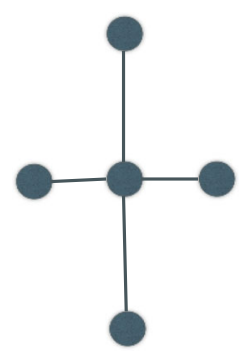

Star

Fig. 1 Examples of network architectures

the geodesic distance between $i_{0}$ and $i_{m}$ as the number of links in this shortest path. We say that the network $g$ is connected if for each pair of nodes there exists a connecting path.

\subsubsection{Sub-networks and Components}

A sub-network $h \subset g$ is a network with set of nodes $S \subseteq N$ and such that $i j \in h \rightarrow i j \in g$. For any subset $S \subset N$ we define the restriction of $g$ to $S$ as the sub-network with set of nodes $S$ and with the links in $g$ that only involve nodes in $S$. The restriction of $g$ to $S$ is denoted by $\left.g\right|_{S}$. We say that the subset of nodes $S \subset N$ is connected in $g$ if $\left.g\right|_{S}$ is connected. A maximal connected sub-network of $g$ is called a component of $g$. By definition, a component $h$ of $g$ is such that no link is present in $g$ between nodes in the component and nodes outside the component. Each component $h \subset g$ identifies a subset of nodes $N(h) \subseteq N$; it is clear that $N(h) \cap N\left(h^{\prime}\right)=\emptyset$ and that $\bigcup_{h} N(h)=N$. The partition of $N$ defined by all subsets $N(h)$ is denoted by $\pi(g)$. So if, for instance, $g=\{12,23,45\}$, then there are two components, $h_{1}=\{12,23\}$ and $h_{2}=\{45\}$, and $\pi(g)=\{\{123\},\{45\}\}$.

\subsection{Representing Networks}

Networks are mathematically complex structures. Yet, we can capture some basic structural properties and compare different networks by looking at simple indices, whose qualitative features are briefly described below.

\subsubsection{Connectivity and Cohesion}

A first basic question is how connected or dense a network is. A first, rough measure is given by the number of links in the network; to be able to compare networks of different sizes, one looks at the average degree; that is, the average number of neighbours nodes have. It is clear that networks with similar number of nodes may present very different topologies, which we would naturally associate with different measures of density. For instance, one may be interested in how far any randomly picked pair of nodes is in the network-the average distance. A different type of information is given by the diameter of a network, the maximal geodesic distance between any two nodes. If for instance, the diameter of $g$ is 6 , it means that it takes at most 6 steps to go from any node to any other node in the network.

Another related feature is network cohesiveness, which aims at capturing how firmly together a group of nodes is in the network. Obviously, adding links to a given network can only increase any reasonable measure of cohesiveness. This is, of course, very different from 
saying that a larger number of links means that a network is more cohesive. To make things clear, consider a star network and a circle. In the star, the removal of a single node is enough to break up the network in two separate components. In the circle, it takes at least two nodes to do it. This can be interpreted as a higher cohesiveness of the circle relative to the star. In general, we can define a cut-set for the connected network $g$ as a set of nodes that, if removed from $g$, would break up $g$ into two or more components. The minimal cardinality of cut-sets of $g$ is the smallest number of nodes that would suffice to break up connectedness of $g$. A common measure of cohesiveness for $g$ is then the cardinality of the smallest cut-set. A similar measure can be applied to any connected subgraph of $g$, measuring therefore how cohesive a sub-group of nodes is in $g$. A celebrated result in graph theory states that if $k$ is such minimal size for $g$, then there exist, for any pair of nodes, at least $k$ connecting paths with empty intersection. This property relates this measure of cohesiveness with a measure of diversification of communication flows between agents in the network. One may also be interested in the vulnerability of a network to the removal of subsets of nodes that are themselves connected in the network. This is an important issue, for instance, if the possibility of such removal is somehow related to some flow of information between nodes in the subset. We can then define a connected cut-set for $\mathrm{g}$ as a cut-set which is internally connected in $\mathrm{g}$. Consistently, we can redefine a measure of cohesiveness based on minimal connected cut-sets (see Sects. 3.3 and 3.4 for applications of these concepts).

\subsubsection{Clustering}

Within a network, two neighbours of a given node may or may not be themselves neighbours. When they are, they "close" the triangle of relationships by forming a "cluster". The degree of clustering may vary greatly across networks, depending on the nature of the relations described by links. In a hierarchical organization, for instance, clustering is very low, while in friendships clustering tend to be quite high, since common friends often become friends themselves. A measure of how clustered a network is looks at all the potential triangles in the networks (a node with two neighbours) and counts the fraction of times that such triangles are actually closed; a slightly different measure takes this fraction for each node in the network, and then averages across all nodes.

\subsubsection{Modularity}

Modularity measures quantify the extent to which the network is partitioned into separate subgroups of nodes, which should be considered as "communities" with little inter-connections and dense intra-connections. These measures (see Scott 2000, pp. 126-145, for a discussion) involve procedures of hierarchical clustering. The degree of network modularity is important, for instance, for the consequences, in terms of aggregate diffusion, of shocks that affect a single community of nodes, or individual nodes. As Golub and Jackson (2012) have shown, homophily and assortative matching, by inducing strong modularity in social ties, can slow down the process of diffusion of information within the network. Hence, local differences tend to persist and society fails to converge to a uniform set of beliefs.

\subsubsection{Centrality}

Nodes in a network may have different degrees of "importance" in connecting other nodes. For instance, a node may be critical in the sense that by removing it from the network, the 
other nodes would split into two or more components. Or a node may be important because many of the shortest paths that connect the other nodes pass through that single node. Or, still, because it is very close to all other nodes in the network, or to the most important nodes in the network.

Centrality indices have the scope of formally quantifying the importance of nodes in the network. A first basic way to think of centrality is to simply consider how many connections a node has - that is, taking a node's degree (possibly normalized by the total number of nodes if one wishes to compare centrality in different networks). Other notions of centrality make use of more global information about the position of nodes in the network. Closeness centrality measures how close a node is to all other nodes in the network, and is given by the inverse of the sum of a node's distances from all other nodes. Betweeness centrality measures how important a node is in efficiently connecting other nodes in the network; for a given node $i$ this index is given by the fraction of shortest paths between any two nodes $k$ and $j$ that go through node $i$. Eigenvalue centrality accounts for the type of connections that a node has in the network; it is based on the (recursive) idea that central nodes are those connected to other central nodes. Finally, Bonacich centrality counts all walks that depart from a given node in the network, discounting longer walks by an exponential factor.

Recent work on strategic behaviour in networks has shown that centrality correlates with agents' behaviour when actions exert local peer effects (see Ballester et al. 2006). This is due to the large exposure of central agents to the positive feedbacks coming from peer effects. When actions also generate local negative externalities in the form, for instance, of passive smoke or transboundary pollution, the relation between centrality and behaviour becomes more complex, and more intense behaviour can move towards the periphery of the network (see Currarini et al. 2014 and, for empirical evidence on smoking, Christakis and Fowler 2002).

\subsubsection{Degree Distributions}

While centrality describes features of single nodes as a function of the whole network, other measures aim at capturing features of the overall distribution of links in the network. The degree distribution of a network provides information about the fraction of nodes that have any given degree in the network. Mathematically, it associates each possible degree $d$ (from 0 to $n-1$, where $n$ is the total number of nodes) with the fraction of nodes with degree $d$ in the network under consideration. It must be noted that although the degree distribution provides useful information on how evenly distributed connections are in a network (whether, for instance, the network has a considerable fraction of nodes that act as hubs and of nodes that are poorly connected or, alternatively, all nodes have more or less the same degree), this measure is silent about other characteristics such as clustering. Figure 2 gives an example of two networks with the same degree distribution (degenerate, with all agents having degree of 2), but quite different architectures in terms of connectivity and clustering.

A benchmark degree distribution is the Poisson distribution, which approximates the expected degree distribution in a purely random network where each link forms with the same given exogenous probability. The fraction of nodes with degree $d$ under the Poisson distribution is $P(d)=\mu^{d} \frac{e^{-\mu}}{d !}$. Real world networks tend to violate independence and uniform randomness in the formation of links, and often exhibit scale free (or power law) distributions, characterized by fatter lower and upper tails compared to the Poisson. Under a scale free distribution, the fraction of agents with degree $d$ is given by $P(d)=c d^{-\gamma}$ (see Fig. 3). The parameter $c$ shifts the expected degree of the distribution, while the parameter $\gamma$ affects the curvature (smaller values generate fatter tails). As it can be easily checked, the 
Fig. 2 Clustering and degree distributions

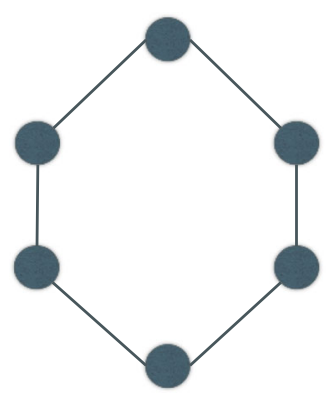

(i)

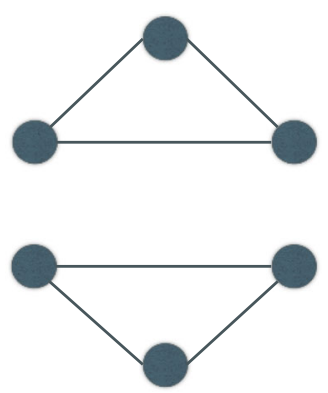

(ii)

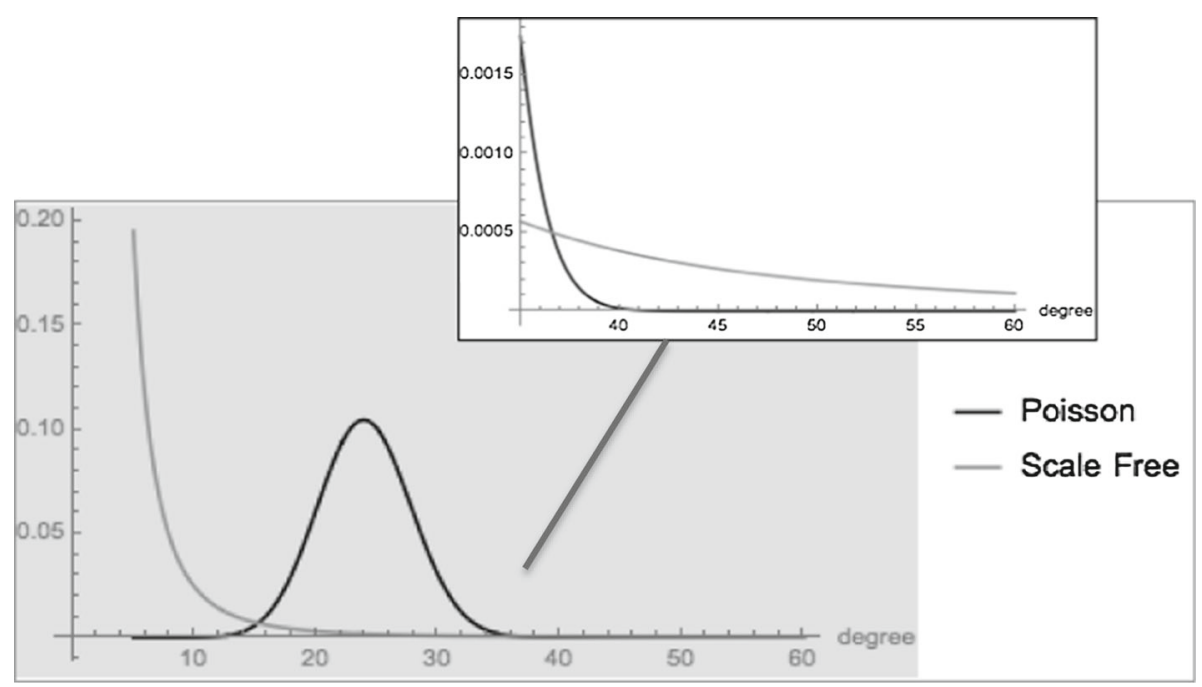

Fig. 3 Poisson and scale free distributions for a network with 60 nodes and average degree of 24. Parameters are $\mathrm{c}=24.4, \gamma=3, \mu=24$. A detailed picture of the upper tails is provided

relative frequency of two different degrees is invariant to the multiplication of both degrees by a common factor (or scale), from which the term "scale free". Such fat tails are generated in models of growing random network where more connected nodes face better chances to form further links with newly born nodes (as in the preferential attachment model by Barabasi 1999).

\subsection{Welfare, Behaviour and Network Formation}

\subsubsection{Network Games, Allocation Rules and Efficient Networks}

The patterns of social interaction are likely to affect agents' behaviour, aggregate welfare and welfare distribution. The traditional models of game theory, in which every agent typically interacts with everyone else, have been extended to encompass the structure of local interactions described by the network. In particular, in graphical games, agents are assumed to only interact with their neighbours in the network, whose actions directly enter their payoff function. However, equilibrium feedbacks are present also between agents who are only indirectly 
connected in the network, and each agent's equilibrium behaviour ends up depending on the entire architecture rather than only on her neighbourhood. Two broad classes of graphical games are: (i) games with strategic complements, where an agent's incentives to act increase with the number (or the share) of neighbours taking the action; (ii) games with strategic substitutes, where incentives to act decrease with the number (or share) of neighbours taking the action. Strategic complements well describe settings where conformism, imitation or other economic mechanisms correlate agents' behaviour with their neighbours'; substitutes represent problems where incentives to free ride are present, and agents substitute their own (costly) action with their neighbours'.

In large networks, agents may have limited knowledge of the overall architecture beyond their neighbour-hood. The class of network games, studied in Galeotti et al. (2010), captures this incomplete information aspect by assuming that only the overall degree distribution of the network is common knowledge, and each agent privately knows her own degree, and formulates expectations about her neighbours' degrees and behaviour. Within this framework, it is possible to draw sharp conclusions about the implications of changes in the network's topology on agents' behaviour in the classes of games with strategic complements and substitutes.

Using a reduced form approach in line with the cooperative games tradition, we can associate with each network $g$ a value function $v$ expressing the total welfare generated by agents in the network. The real number $v(g)$ can be thought as the sum of agents payoffs in a game played on $g$, or as the social "pie" that is generated in $g$ and that must be distributed among agents. Individual payoffs are represented by an allocation rule $a(v, g)$, a vectorvalued function mapping each economic problem (a pair $v, g$ ) into a distribution of the value $v(g)$. A network $g^{*}$ is said to be efficient with respect to $v$ if it maximizes the size of the pie to be distributed: $g^{*}=\operatorname{argmax}_{g} v(g)$.

\subsubsection{Link Formation, Stability and Efficiency}

The way in which the allocation rule $a(v, g)$ distributes the total pie among agents determines agents' incentives to form and sever links. For instance, agent $i$ (node $i$ ) in network $g$ will have an incentive to form the link $i j \notin g$ if her payoff, as determined by the rule $a(v, g)$, would increase in $g+i j$ compared to $g$. Any notion of stability of a network refers to such incentives, and is therefore defined with respect to the pair $(v, a)$. Depending on agents' strategic possibilities to revise their links we obtain various notions of stability.

One first important issue is whether agents can form links without the consent of their perspective partners. This modelling choice clearly depends on the specific economic problem one has in mind, and in particular on whether links are directed or undirected. If links represent literature citations or Internet page referrals, unilateral link formation is an appropriate assumption. Mutual consent is instead required in international environmental agreements, information sharing, friendships, insurance, market agreements, co-authorship, and in many other socio-economic applications.

A second issue is the extent to which agents are able to coordinate their decision to revise links. When links can be formed unilaterally, stability can be defined by directly applying the Nash equilibrium to a suitably defined link formation game. Coordination is instead a crucial issue when mutual consent is required to form a link, since individual actions are not capable of adding links to a network. The notion of pairwise stability (Jackson and Wolinsky 1996) assumes that agents can coordinate to form a profitable link: a stable network obtains when no pair of agents wishes to form a new link, and no agent wishes to (unilaterally) sever an existing link. Note that pairwise stability cannot be derived as the Nash equilibrium of a 
suitably defined link formation game, since: (i) agents can only sever one of their existing links, and (ii) pairs of agents can jointly deviate from a network by forming a new link. The notion of Nash-pairwise stability, allowing both the coordinated objection of pairs of agents and the severance of any number of an agent's own connections, is instead a refinement of the Nash equilibrium. The even more demanding notion of strong stability (Jackson and van den Nouweland 2005), assumes that any subset of agents can coordinate in the joint revision of their links, and possesses similar features to the strong Nash equilibrium of games in strategic form.

Since an agent's decisions to add or sever links potentially affect all other agents in the network (the so called network externalities), decentralized linking decisions are likely to lead to inefficient networks from a social point of view. Jackson and Wolinsky (1996) have shown that network formation suffers indeed from a general tension between stability and efficiency. They show that no allocation rule $a(v, g)$ that satisfies natural symmetry and anonymity properties guarantees that the efficient network will be pairwise stable. Given the limited amount of coordination required by the pairwise stability notion, this result highlights a serious inconsistency between private and social incentives in network formation. Other contributions have proposed ways to overcome this tension, focusing on either mechanism design approaches (Dutta and Mutuswami 1997), or on Coase-like bargaining procedures (Currarini and Morelli 2000), or still on general transfers schemes (Bloch and Jackson 2007). When spillovers are present across components (a relevant case for environmental problems, where agents benefit and suffer from actions taken by other disconnected agents), efficiency generally requires the use of contingent transfers, which subsidize the formation or the deletion of those links that are responsible for the spillovers (see Bloch and Jackson 2007).

\section{Mapping the Linkages Between Network Economics and the Environment}

\subsection{Diffusion and Evolution in Networks}

Many aspects of environmental economics and resource management problems are intrinsically dynamic. For example, natural resources are often stocks whose laws of motion are affected by both natural factors and human behaviours. The opinions, beliefs and incentives that determine environmental behaviours tend to evolve in time, and to spread through society according to dynamics that depend on the patterns of social connections.

In this chapter we discuss the role of networks in our understanding of such dynamics, and provide a specific example of how networks have fruitfully enriched models of environmental behaviour. Although we treat "diffusion" and "evolution" separately, these phenomena are strictly related, and models are often formally equivalent.

\subsubsection{Diffusion of Green Technologies}

Diffusion is defined by Rogers (1995) as "the process by which an innovation is communicated through certain channels over time among the members of a social system". The key terms in this definition are the novelty of the piece of information to be transmitted, and the role of social channels for this spread. Beliefs, infections, fashions and various kinds of behaviors spread through society in very much the same way as information does. Understanding the diffusion process is a central issue in environmental sciences to the extent that what spreads in society has a potential effect on natural resources and their management. This is the case, 


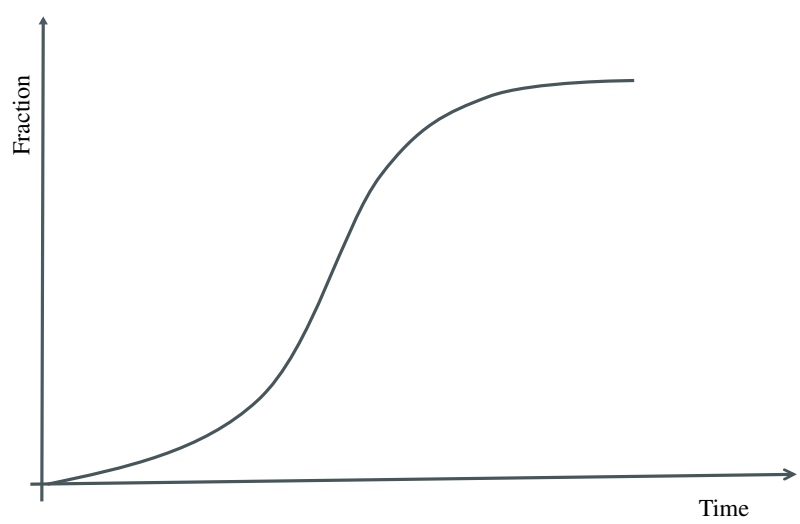

Fig. 4 An example of S-shaped diffusion curve

for instance, for the adoption of innovative technologies by firms (Conley and Udry 2001), of green behaviour by consumers, as well as cooperative behaviour by signatories of an international environmental agreement (Heal and Kunreuther 2012).

The early models on diffusion (see Bass 1969) neglect the local nature of interaction, and focus on the overall "rate of adoption" in society, whose dynamics are governed by the overall ratio of adopters and of non adopters at any point in time. The key insight from the Bass model is that diffusion follows a nonlinear trend, with first a fast acceleration and a subsequent slowdown (Fig. 4, Young 2009).

Although the Bass model contains no "micro-foundation" for the dynamics of the rate of adoption, similar nonlinear patterns of diffusion are obtained in models where agents optimally decide whether to adopt as a function of how many adopters are present in societies. Such models are built on the idea of strategic complementarities, where the incentives to adopt increase with the fraction of other agents adopting the innovation. Depending on the context, many definitions have been given to the idea that other people's actions can reinforce one's own choices: 'bandwagon effects' in fashion-oriented behaviour (Leibenstein 1950), individuals' adoption thresholds (Granovetter 1978), entrapment (Dixit 2003), network externalities, social reinforcement, cascades (Watts 2002), tipping (Gladwell 2000) and "positive feedback trading" in finance (Barberis and Shleifer 2003), among others. Empirical work relating to environmental problems has established the relevance of the S-shaped curve for the diffusion and adoption of new technologies. Ryan and Gross (1943) and Griliches (1957) demonstrated that the adoption of hybrid corn seeds among Iowa farmers follow the pattern presented in Fig. 4. More recently, Weir and Knight (2004) find a significant role of schooling, mediated by social networks, in the adoption and diffusion of innovations using data from Ethiopia. Specifically, they suggest that literate farmers are early adopters of new farming practices as well as quick at imitating innovations by others, while illiterate farmers tend to be second-movers and eventually adopt the practices of the innovators.

The role of complementarities for diffusion is also central to works that study the presence of tipping point for the adoption of climate policies by the international community. Heal and Kunreuther (2012) offer illustrative evidence on the role of early adopters (i.e. those located at the left x-axis corner in Fig. 4) in triggering a global shift from damaging pollutants' usage to greener alternatives. The first one concerns the adoption of unleaded gasoline in replacement of leaded gasoline; here the unilateral adoption by the United States meant that the subsequent adoption's costs for other countries was confined to modifying refinery capacity, since motor 
industries exporting to the U.S. had to transition to lead-free fuel immediately after the move. Thanks to these reduced costs for the followers, the new technology spread quickly worldwide. Another example refers to phasing out chlorofluorocarbons (CFCs), a spectacular achievement of the Montreal Protocol on Substances that Deplete the Ozone Layer. ${ }^{1}$ In this case, the U.S. decision to sign the Montreal Protocol hinged on a technological innovation by Du Pont, the world's largest producer of CFCs, allowing the company to gain from elimination of CFCs. Again, strategic complementary led most countries to phase-out ozone-depleting chemicals.

While absent from these early models of diffusion, the role of social contacts (the "channels" in Rogers 1995's definition) in determining the final outcome of diffusion is little disputable. For instance, the probability that a substantial fraction of the population will get infected by a virus, initially affecting only a small subset of agents, will largely depend on how densely connected the network is, on the shape of the degree distribution, and on how these features relate to the specifics of the infection (how aggressive and contagious it is, how costly immunizing part of society is, etc...). Similarly, a farmer's incentive to adopt a new technology will depend on how many of his neighbors have adopted it, and probably on how profitable the adoption turned out to be.

The theoretical effort devoted to the extension of the basic aggregate models of diffusion to encompass the role of networks has produced a vast and growing literature. This literature tries to address issues such as the effect of different network topologies on the speed of diffusion, the role of mediators and perpetrators, the effect of assortative matching (homophily) on the spread of information, the role of the costs of diffusion.

In the epidemiology literature, the two main benchmarks are the SIR (Susceptible, Infected, Recovered) and the SIS (Susceptible, Infected, Susceptible) models of diffusion. In the SIS model, agents are born healthy (susceptible), get infected with some probability and with some probability recover, after which they become susceptible of infection again. Here the network topology affects the probability that an agent gets infected; this is proportional to its degree and to the probability that one of his neighbours is infected (and also to the rate of disease transmission, measuring how infectious the disease is but not depending on the network structure). Both the speed of convergence to steady state with non-zero infection and the overall social extent of the infection depend on the degree distribution of the network, and on how this topology relates to the rate of transmission (see Jackson 2008).

Examining the effect of changes in the degree distribution provides an instructive example of what one can learn by explicitly modelling the network. First, a first order stochastic dominance shift in the degree distribution (larger average degree) always increases the speed of diffusion and the steady state share of infected agents. This result has a very natural intuition: more connections result in more intense social interaction and in a faster spread of the disease. Second, a shift of the second order stochastic dominance type (larger fractions of agents with very small and with very large degrees) has ambiguous effects: while a higher fraction of poorly connected agents should slow down diffusion, a larger fraction of very well connected agents should speed it up. The net effect depends on the topology of the network, and on how this topology combines with the degree of infectiousness of the disease. When infection rates are very high overall, a spread of the degree distribution results in a decrease in diffusion, and vice versa. An intuition for this result comes from the non-linear relation between a node's degree and its probability of infection: already high infection rates are little sensitive to increases in a node's degree, simply because infection rates are bounded above.

1 As of September 2013, 197 countries have ratified the Protocol, banning the production of chlorofluorocarbons, halons, and other ozone-depleting chemicals. 
So, when infection rates are high, the effect of increasing the number of very connected nodes is small and dominated by the effect of increasing the number of little connected nodes. By a specular arguments, when the infection is little aggressive a spread in the degree distribution increases the speed of diffusion, as the effect of more nodes with large degree dominates the effect of more nodes with very small degree.

Interestingly, similar insights characterize problems with strategic complementarities, where the probability of taking an action increases with the share (or number) of neighbours taking that same action. Here as well more connections imply faster diffusion, and the effect of a mean preserving spread in the degree distribution is ambiguous. Faster diffusion occurs when the incentives to adopt are very sensitive to the degree of an agent-a similar condition to the one for the SIS model, where the rate of transmission had to be very sensitive to the degree. A notable difference with respect to the SIS model is that conditions for large scale spread apply to the size of the initial adopters, which has to exceed a given threshold in order for diffusion to kick off (an intuitive consequence of complementarities).

\subsubsection{Evolution of Cooperation in Networks}

Cooperative behaviour and social dilemmas are of great importance in environmental economics. The stylized and tractable framework of the Prisoner's Dilemma has spurred an immense literature trying to assess the potential of socio-economic systems to overcome the inefficiencies of private incentives. ${ }^{2}$ In environmental problems, this relates to the ability of a system to preserve exhaustible natural resources, manage commons and produce public goods. Since cooperative behaviour (especially within groups) seems to be at the root of successful evolution, despite the fact that co-operators are themselves, as individuals, under evolutionary pressures, the emergence of cooperation has traditionally been of interest to evolutionary biologists. Evolutionary models have then been established as the standard paradigm to study cooperation. A fundamental question in this body of research is under what conditions cooperation would prevail as a product of evolutionary dynamics. A useful starting point is the early result showing that when social interaction is unstructured (the so-called well-mixed matching model, where every agent may interact with every other agent), natural selection increases the relative abundance of defectors and drives cooperators to extinction (see, for instance, Hofbauer and Sigmund 1998; Nowak et al. 2004). Cooperation is therefore unviable in the long run.

Researchers have then focused on various mechanisms that may generate either partial or widespread long run cooperation. One of such mechanism is of direct interest to the present survey, as it focuses on the role of local (or "spatial" or "targeted") interaction. ${ }^{3}$ The general idea is that agents do not observe, or interact with, all other agents in the system, but only with a restricted set of neighbours. In this framework, local interaction determines both individual payoffs (via the game being played locally) and the (myopic) payoff comparisons that drive the revision mechanism. The main finding of this literature is that within such structured populations (described by an incomplete network), the outcome of evolutionary games can be very different from the one of the well-mixed case. The early work of Nowak and May (1992) shows that when local interaction takes the form of a two dimensional lattice, chaotic dynamics may persist in the long run, with both cooperators and defectors present in non vanishing portion of society. In general, the possibility that cooperation occurs in the long

2 Other social games that have been studied in the evolutionary literature are the "stage-hunt" and the "snowdrift" games, where dominant strategies are absent and dynamics select one out of multiple equilibria.

3 These also include direct reciprocity, indirect reciprocity, kin selection and multi-level selection. 
run has been shown to depend on depend on whether the benefit-to-cost ratio of cooperation exceeds the average number of interactions (Ohtsuki et al. 2006). Moreover, "diversity" in the degree distribution has been shown to help promote cooperation. In particular, various studies confirm that scale free distributions (i.e., networks in which very connected and very little connected agents abound compared to uniform random assortment) are able to sustain partial cooperation in the long run, independently of the underlying game being played ${ }^{4}$ (Alonso-Sanz 2009; Santos and Pacheco 2005; Santos et al. 2006, 2012; Rand et al. 2014).

A very recent and insightful line of research is now looking at the effects of the coevolution of behaviour and interaction structures. In fact, it seems reasonable to assume that not only behaviour is affected by the structure of social contacts, but also that this structure may change in response to experienced behaviour. Agents may in fact try to form or sever links in order to take advantage of more profitable partners, or to mimic the social contacts of successful neighbours, or just because their preferences may change due to past interactions. Early attempts to study the coevolution of network and behaviour have simply assumed that the network itself evolves according to its own evolutionary dynamics. This approach is found also in more recent papers (see, for instance, Ebel and Bornholdt 2002a; Szolnoki and Perc 2009c).

A richer approach fully encompasses the feedback between behaviour and social structure, and allows the network dynamics to depend on past strategies (see Ebel and Bornholdt 2002b; Chen et al. 2008; Gräser et al. 2009). Skyrms and Pemantle (2000) first suggested how considering the dynamic of the network, as it generates from agents' linking decisions, can have substantial effects on the evolution of cooperation compared to models with a fixed network. Interestingly, a careful examination of these feedbacks has lead to results that are at odds with previously shared wisdom on what type of social structure promotes cooperation. In particular, Hanaki et al. (2007) have shown how the survival of a cooperating population requires sparse networks, little local clustering, and the possibility that agents unilaterally cut unprofitable ties. This findings are at odds with the prior wisdom that cooperation would develope in closed and dense communities of cooperators (see Axelrod 1984; Coleman 1988). Similar insights underly the results by Santos et al. (2012), showing that structures that promote cooperation, exhibiting enough diversity in the degree distribution, can arise by means of agents' voluntary link revision.

No work to our knowledge has studied the coevolution of the social structure, behavior and agents' preferences (such as, for instance, prejudice against diversity or social awareness). This seems to be a promising direction of research in environmental economics, where environmentally aware behavior by consumers tends to diffuse according to social relations, and is also likely to shape (and be itself shaped) by the formation of new social ties.

\subsubsection{Coevolution of Behavior and Natural Resources}

We end this section with a specific example of what additional insights are obtained by modelling network interaction in problems of diffusion of behaviour. In particular we consider the evolution of cooperative behaviour in resource harvesting. Tavoni et al. (2012) and Lade et al. (2013) explored the effectiveness of social sanctioning of resource overuse in promoting sustainable extraction. Two types of agents, norm-following co-operators (C) limiting their resource use to the socially optimal amount, and defectors (D) who extract above the

\footnotetext{
4 The two main metaphors for the tension between private and collective incentives are the Prisoners' Dilemma (in which defecting is a dominant strategy) and the Snowdrift Game (also known as "Chicken Game", in which cooperation is a best response to defection and viceversa). For the Snowdrift Game, Hauert and Doebeli (2004) have shown that local interaction may inhibit co-operation rather than promoting it.
} 


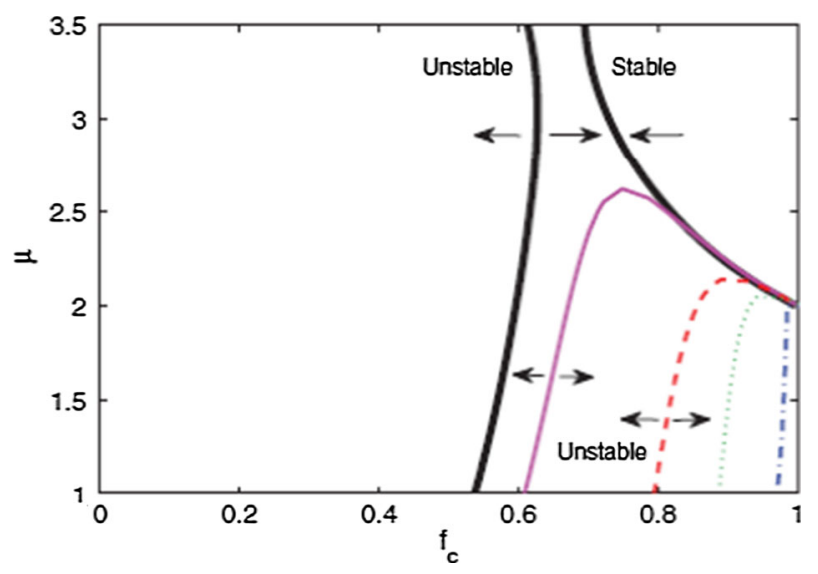

Fig. 5 Co-existence of co-operators and defectors in a well-mixed population (thick solid curve) and in regular networks with decreasing average degree. $\mu \in(1,3.5)$ is the degree of defection relative to $\mathrm{C}$, and $f_{c}$ is the frequency of C-types. Source Chung et al. (2013)

sustainable level, interact in a well-mixed population (i.e. absent a network structure). Either type, when randomly matched with a fellow user of the shared resource, updates his or her strategy based on utility differences, according to the replicator dynamics. A key feature of this model is the explicit consideration of resource dynamics, which are coupled with behavioural dynamics. Payoffs from harvesting vary depending on the composition of the population: the higher the share of defectors, the more depleted the resource and the less effective the sanctions. The results of this a-spatial model, where everyone interacts with everyone else, are displayed in Fig. 5 for varying degrees of the parameter $\mu$, which captures the extent of norm violation. ${ }^{5}$ Three regimes of stationary state of the evolutionary dynamics obtain: (i) the defector equilibrium (when the dynamics tend to the left of the figure); (ii) the co-operator equilibrium (on the right hand side of the figure); and (iii) the mixed equilibrium where both $\mathrm{C}$ and $\mathrm{D}$ coexist.

Chung et al. (2013) have enriched this model with an explicit account of the structure of local interactions, formalized by a social network. The thinner lines in Fig. 5 show results for a model where individuals only observe agents in their neighbourhood. Here, the effect of the sanctions imposed on a norm violator is assumed to depend exclusively on the fraction of cooperators in the defector's neighbourhood. ${ }^{6}$ The figure refers to regular connected networks of 50 nodes, with degrees of $k=40$ (solid curve), $k=20$ (dashed curve), $k=10$ (dotted curve) and $k=2$ (dash-dotted curve). We observe that as the common degree declines, the basin of attraction of the co-operator equilibrium shrinks, paving the way for a 'tragedy of the commons'.

Interestingly, the joint account of behaviour and resource dynamics generates predictions that are somewhat at odds with those obtained in models where resource dynamics are absent. While in such models long run cooperation requires sparse networks and low clustering or, at least, the presence of many little connected agents (see the previous section for a discus-

5 The arrows in the figure indicate the direction in which the composition of the population evolves, so that one can determine which equilibrium obtains for a given initial share of co-operators (and the level of defection by norm-violators).

6 This is in contrast to the well-mixed population, where all defectors are subject to the same amount of ostracism. 
sion), here stable long run cooperation is consistent only with highly connected networks. Further research should qualify such predictions for non-regular networks, such as scale-free networks, where cooperation has been shown to be consistent with long run evolutionary selection.

\subsection{Access and Use of Natural Resources}

The access and distribution of natural resources often entails the use of networked infrastructures and markets. This is the case, for instance, of irrigation water and natural gas. In these examples, the cost and benefits from the use of the resource is determined by the pattern of canals and pipelines through which this is sourced and distributed. The efficient use of the resource calls for agreements and contracts between the nodes of the network, whose gains and benefits are determined through complex bargaining processes. The network itself is, to some extent, flexible, as new links can be created and existing links destroyed in pursue of larger profits. The incentives to form or delete a link may well not align with social incentives, as the formation only requires the consent of the two interested nodes, and the deletion often only requires the consent of one of the interested nodes. Due to network externalities, inefficient networks may result from the decentralized formation of links, and a general tension between efficiency and stability has been recognized in early works of network economics (see Jackson and Wolinsky 1996). Two issues seem to be of prominent interest for the application of network economics to natural resources: how players will share the gains from cooperation through bargaining, and how this will affect, and be affected, by the degree of flexibility of the network and the incentives to form and delete links.

In this section we discuss these issues using, as an illustrative example, the case of the Eurasian natural gas pipelines. Eurasian gas accounts for $40 \%$ of EU gas imports, and most of these imports transit through Belarus or Ukraine, both importing gas from the Russian Federation.

A striking example of the stark consequences of failures in the bargaining process is provided by the 2009 crisis, where the disagreement on gas prices and fees led to interruptions

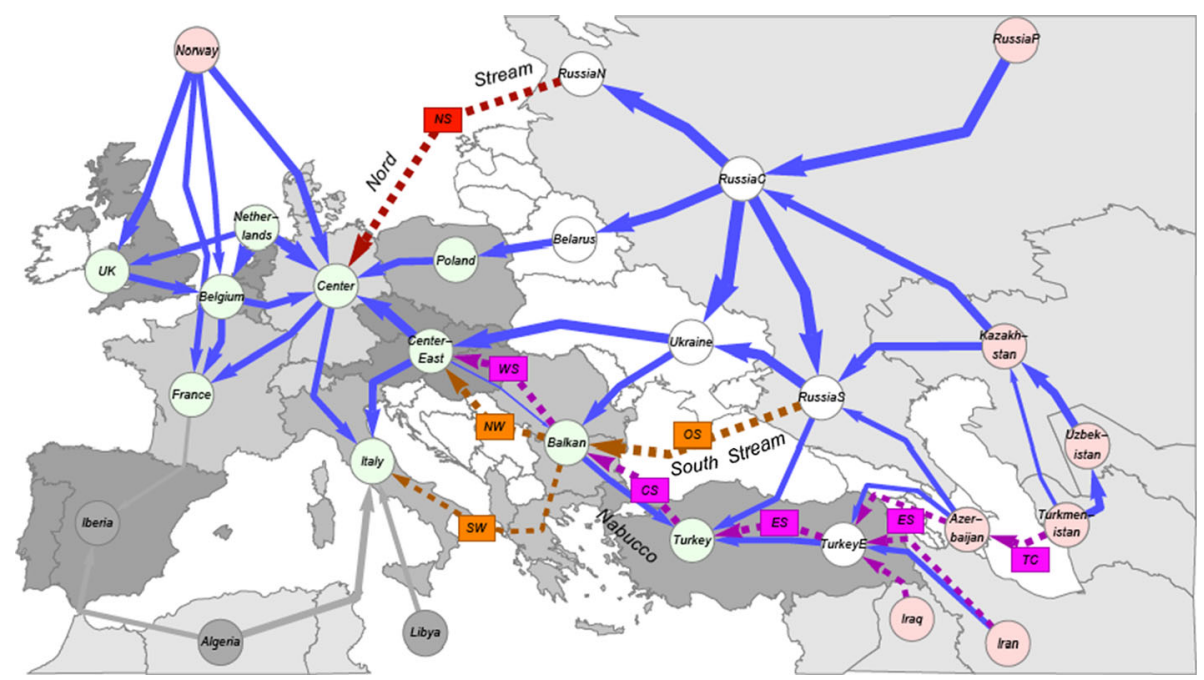

Fig. 6 Eurasian pipeline gas network. Source Hubert and Cobanli 
of supply through Ukraine for several weeks (see Hubert and Cobanli 2012, Fig. 6). Such retaliatory behaviour can be viewed as an attempt to build-up bargaining power out of strategic and vital positions in the transmission network. In general, players' bargaining power (and, with it, their final payoff) will depend on their position in the pipeline network, together with other factors such as market size, production capacity and international power. Recent contributions in network theory have provided a framework to study bargaining processes among agents located on a network. These are mainly buyer-seller networks, in which a player bargaining power depends, in a complex manner, on her connections to other nodes on other side of the market, and on the connections of these nodes. However, distribution networks generally have a more complex structure than buyer-seller networks. Specifically, they are characterized by directed links and present strong heterogeneities among players; incorporating these features within a fully-fledged non cooperative bargaining model is a challenging, yet necessary task for future research.

Adopting an alternative approach, based on cooperative game theory, Hubert and Cobanli (2012) have studied the specific problem of Eurasian gas pipelines network. This approach relies on a variation of the Shapely Value for games with a communication structure, that is, games where agents' cooperation possibilities are described by a network. This variant, first proposed by Myerson (1977), is based on a description of coalitional values that take into account the limits to cooperation imposed by the fixed network structure. Using the notation developed in Sect. 2, we let $\left.g\right|_{S}$ be the sub-network obtained by only considering nodes in $S$ and those links for which at least one of the involved nodes belongs to $S$. Let also $C\left(\left.g\right|_{S}\right)$ be the set of components of $\left.g\right|_{S}$, and let $\pi\left(\left.g\right|_{S}\right)$ be the partition obtained by considering the set of nodes of the components in $C\left(\left.g\right|_{S}\right)$. Given a primitive characteristic function $v(S)$ describing the payoff possibilities of each coalition $S$, we can define the new value function $v_{g}=\sum_{B \in \pi(g \mid s)} v(B)$. This function captures the fact that players without links in $g$ are not able to coordinate their actions unless indirectly connected by other players who transmit the necessary information. So, the coalition $S$ is only able to generate a value equal to the sum of values generated by its connected components. The marginal contributions that enter the computation of the Shapley value are, of course, affected by the network. In particular, players who are vital for many connected components, end up having very large marginal contributions and, therefore, a large Shapley value. This, in turn, implies that players who act as connectors in the network will be allocated a relatively large share of the aggregate payoff. Within the context of the Eurasia pipeline network, the resulting allocation rule is such that those countries that, if removed from the network, would impede the flow of gas from sources to users, such as Belarus and Ukraine, have a strong bargaining power.

The outlined relation between the network architecture and players' bargaining power can help interpret recent developments in the (planned) infrastructures of gas distribution. These include: (i) the offshore twin-pipeline Nord Stream, which establishes a direct link between Russia and Germany through the Baltic Sea; (ii) the South Stream pipeline, providing a direct connection between Russia and Bulgaria, from where gas should flow to Central Europe, Italy and Turkey; and (iii) the Nabucco project which should open a corridor through Turkey, thus connecting Europe to new suppliers in the Middle East and the Caspian region. If implemented, these projects would considerably weaken the bargaining position of Belarus and Ukraine, reshaping the power along the network at the advantage of Russia and Europe. In terms of network economics, the very fact that these projects are being planned or undertaken suggest that the current configuration of pipelines does not constitute a "pairwise stable" network architecture (see Jackson and Wolinsky 1996). This notion of stability would in fact require that no pair of nodes has an incentive to bear the cost of a new link, and that no node find it profitable to cut any of its links. As in the case of the newly planned pipelines, 
such incentives are determined by the expectation of a new payoff allocation following the creation or deletion of a link. This makes clear that a better understanding of the economics and strategy of networked resources would call for an analysis of network formation, itself based on a careful assessment of incentives to form and sever links, of the associated costs and gains in bargaining power, and of the consequences for the system as a whole. This seems to be a challenging and exciting area for future research.

\subsection{Common-pool Resource Management and Governance}

The collective management of natural resources is increasingly being recognised as a critical dimension of sustainable development and a key determinant of economic performance, especially in the rural sector of developing economies (Platteau 1991; Baland and Platteau 1996; Ostrom 2003; Ballester et al. 2006; Baland et al. 2006). By its nature, collective action involves interdependency among individuals. For example, the maintenance of an irrigation network requires the stabilization of the rims and the desalting of minor channels across farmers' land. Interdependency, combined with the non-excludable and rival nature of many natural resources, poses significant challenges and raises the question of whether individuals are capable to coordinate their action and successfully manage resources held in common.

The conventional theory of collective action-centred on the powerful metaphor of the tragedy of the commons (Hardin 1968), the Prisoner's Dilemma game, and the free-riding problem (Olson 1965) —offered a pessimistic yet influential answer to this question, that has for long dominated the way in which social scientists thought about shared resources (Ostrom et al. 1999). Over the past decades, however, scholars from different disciplines and backgrounds have shown that the tragedy of the commons is not inevitable, and individuals have the potentials to act collectively. ${ }^{7}$ The recognition that collective action is possible has, in turn, shifted the attention of more recent research towards the question of why collective action emerges and under which conditions it is more likely to succeed or fail. Within this context, a number of structural variables have been identified as critical for the successful management of common-pool resources. These include institutional arrangements concerning monitoring, sanctions and accountability; group characteristics related to size, levels of wealth, and social capital; attributes of the social environment and of the resource system, such as the social cohesion and the networked structure of groups, well-defined boundaries, unpredictability of resource flows, and resource mobility.

While the first two sets of variables-i.e. institutional arrangements and group characteristics - have been studied extensively both theoretically and empirically, our understanding of factors related to resource characteristics and the role of social networks is still relatively limited. Yet the physical complexity of natural resources may have important implications for whether and how users can sustain effective institutions. For example, as water moves through a landscape, hydrological attributes such as quantity, quality, location and timing, are likely to be influenced by land use and vegetation patterns. The interconnected nature of the hydrological cycle, thus, implies that many actors and sectors influence water resources at different geographic scales and administrative levels of governance.

In this section we will discuss recent contributions and possible advances in two of the above aspects of the management of common resources, both calling for a network perspective: multiple commons and multi-level governance in the water sector.

\footnotetext{
7 Examples of cooperative behaviour have been identified in a wide range of contexts. These include the management of fisheries (e.g., Acheson 2003; Singleton 1999), forests (e.g., McKean 1986, 2000; Schoonmaker Freudnberger 1993), pastures (e.g., Gilles et al. 1992; Netting 1981; Nugent and Sanchez 1999), and groundwater resources (e.g., Trawick 2003; Marchiori et al. 2012).
} 
Fig. 7 Two different networks of two cities and two sources ssssources

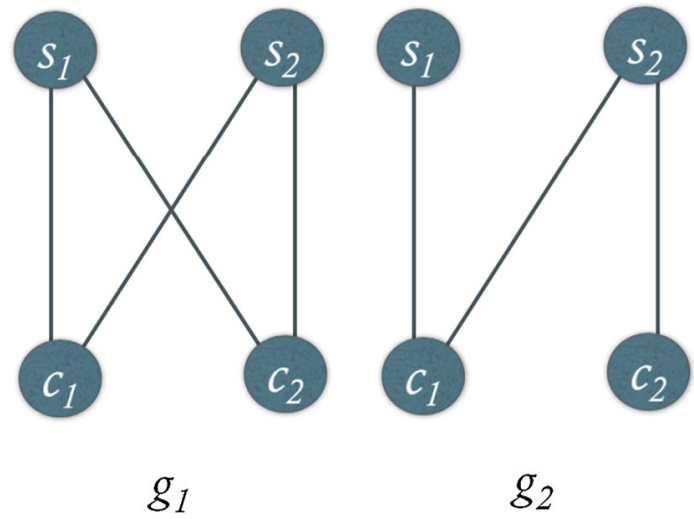

\subsubsection{Networks of Commons}

Most models of commons assume that there exists a single source exploited by many users. In fact, the most representative commons (e.g. forests, pastures, and groundwater resources) are local, but numerous. The multiplicity of sources can raise interesting political and economic questions. For example, the severe drought that affected Spain in 2006-2007 led the government to consider the possibility of transferring water from the north to the south through the construction of new pipelines. This proposal gave rise to a political debate about regional and national sovereignty over water resources, and the potential economic and environmental consequences of water transfers.

A first important step towards the analysis of common-pool resource problems with multiple sources was recently made by Ilkiliç (2011). In this paper, the author considers a situation in which $n$ (water) sources $s_{1}, s_{2}, \ldots, s_{n}$ and $m$ cities $c_{1}, c_{2}, \ldots, c_{m}$ are embedded in a network that links cities with sources. Figure 7 provides two examples of possible network structures in the case of two cities and two sources. The first graph, describes a complete network where each user is linked with both sources, while in the second graph, $c_{2}$ is connected only to $s_{2}$.

The cities receive a value from consumption of the resource, but extraction is costly. Water consumption has linear benefits; extraction costs are convex, and are composed of a private part, which depends quadratically on total extraction by the city, and of a part that is source specific and is subject to a congestion effect. Specifically, city $j$ 's utility takes the following form:

$$
u_{j}\left(Q_{g}\right)=q_{j}-\frac{q_{j}^{2}}{2}-\sum_{s_{i} \in N_{g}\left(c_{j}\right)} q_{i j} q_{i}
$$

where $q_{i j}$ is the amount of water extracted by city $c_{j}$ from source $s_{i}, q_{j}$ is the total amount extracted by $c_{j}$, and $q_{i}$ is the total amount extracted from source $s_{i}$. So, a city's extraction from a given source has a negative cost externality on all other users of that same source. There is therefore no physical externality across sources. In particular, this implies that a model with two sources is different from a model with one source even when all cities are linked to both sources.

Two important issues in this setting concern the non-cooperative extraction levels when users freely decide how much to extract from each source they are connected to, and how these 
levels compare to the socially efficient use of the sources. A first main contribution of the paper is that each city's non-cooperative exploitation of a given source turns out to be proportional to a measure of Bonacich centrality of the link connecting the city to that source. ${ }^{8}$ Consider, for instance, the two cities-two sources case of figure 7. It can be shown that, if the network structure is as in graph $g_{1}$, the link flows at equilibrium are $q_{11}^{*}=q_{21}^{*}=q_{12}^{*}=q_{22}^{*}=0.2$. These levels equate marginal costs across sources for both cities, given the negative cost externality associated with local extraction. Interestingly, these are equivalent to the levels of extraction that would occur if each city was linked to a different source only. So, in the complete network agents avoid the cost inefficiencies by sorting their extraction between the two sources. If the network is incomplete as in $g_{2}$, equilibrium extraction levels at equilibrium are $q_{11}^{*}=0.2857, q_{21}^{*}=0.1429$, and $q_{22}^{*}=0.2857$. In this case, $c_{2}-$ which is now connected only to $s_{2}$-exploits this source more than in the complete network. This, in turn, makes the extractions from $s_{2}$ more costly, leading $c_{1}$ to consume less water from this source and rely relatively more on its exclusive connection $s_{1}$. Hence the absent link between $c_{2}$ and $s_{1}$ harms $c_{2}$ (which is lacking the link) and benefits $c_{1}$ (the city that is connected to both sources).

More generally (and interestingly), in a common-pool resource game with multiple sources, a user's extraction at a source does not only depend on the number of users it shares it with. It also depends on the number of sources that these other users are linked to; and on the number of users those sources are linked to, and so on. That is, the externality spreads through the network and across sources, despite the absence of any physical interdependence across sources.

From a policy perspective, the analysis suggests that disregarding the structure of the network may be misleading, because different structures affect both overall extraction levels and the distribution of the resource across users and sources. Going back to the previous example, the complete network $g_{1}$ leads to relatively higher overall water consumption. However, the incomplete structure $g_{2}$ is such that $s_{2}$ is exploited more severely. This, in turn, may have implications for both the urgency and type of intervention depending on how close to the point of non-recovery is the resource as a whole, and on the ecological and socio-economic importance of different sources within the network.

Turning now to the efficient use of the various sources, the paper first shows that all efficient allocations are characterized by the same aggregate extractions source by source and city by city. More importantly, it turns out that the efficient use of water in a given network is equivalent to the efficient use that would result by partitioning cities and sources into independent "regions". Each region would comprise a subset of cities together with the sources to which these cities have access in the network, and within each region the aggregate water use from each source would be the same as if the region was internally fully connected. This conclusion seems to support a management approach based on the creation of distinct and independent areas of water exploitation, where subsets of cities have exclusive access to a subset of sources.

The paper opens the way to salient and challenging questions concerning the use of networked resources. First, linking cities to sources may be a (costly) policy decision. The crucial question is therefore which networks are socially efficient in this case, and whether agents would indeed form the efficient links in a decentralized link formation game. Efficiency is a property of link formation in the two sided problem studied by Kranton and Minehart (2001), and it would be interesting to see whether those results apply to problems with local

8 Note that while in the traditional model of games on networks, where each node is a player, equilibrium behaviour relates to the Bonacich centrality of nodes (see Ballester et al. 2006), here the equilibrium relates to the Bonacich centrality of links. This is due to the fact that the city-to-source network is bipartite and only the nodes in one of the two independent sets (the cities) are strategic players. 
congestion. Second, many real life instances where multiple commons are relevant present spillovers across sources. We think, for instance of the complex interplay between groundwater and surface water, but also of catchment areas in fisheries. Finally, cities and sources may be heterogeneous, in costs and sizes, and this would naturally induce heterogeneous extraction levels and efficient architectures.

\subsubsection{Multi-level Governance in the Water Sector}

The inherent complexity of water governance is due not only to the interconnectedness of the hydrological cycle, but also to the multiplicity and variety of actors affecting water resources at multiple levels. Responses to water problems are often based on top-down centralised approaches, generally poorly suited to deal with the socio-political and ecological complexities underpinning water use and management (Pretty and Ward 2001; Molle et al. 2007). The emerging new frameworks for governing water see national governments increasingly devolving decision-making responsibility to local authorities and encouraging stakeholders' participation. The underlying rationale is that involving actors at different levels can lead to improved accountability of stakeholders, higher legitimacy of the decisions, and management strategies that are better adapted to local conditions (e.g. Marchiori et al. 2012).

Researchers in environmental policy distinguish between a 'vertical' and a 'horizontal' dimension of governance, where the former refers to the linkages between higher and lower levels of government, including institutional, financial, and informational aspects; the latter refers to cooperative arrangements between a range of public and private actors in the formulation and adoption of development strategies. Recent research has stressed how, by shaping the functioning of such arrangements, social networks can either facilitate or hinder the implementation of and the compliance with environmental regulations. Researchers have argued that social ties of various kinds, ranging from information transmission channels to power relations, social emulation, market interaction, kinship, funding, collaboration etc., can be more important than formal decision making structures for the success of environmental cooperation (see, for instance, Olsson et al. 2008).

In the specific case of water management, using a network approach to assess the effectiveness of policies involves two steps: first, identifying all the actors that directly and indirectly influence the complex process of water resource use; second, mapping their formal and informal relations. Direct influence means that an actor directly modifies water flows through withdrawals and discharge activities, flow control measures and land use. Other actors may exert an indirect influence by affecting the activities of those who use water directly. Think, for example, of a governmental body that provides funding for the construction of a new irrigation scheme.

To date, empirical research applying quantitative network analysis to natural resource governance is still relatively limited. Yet, some valuable insights and hypotheses have started to emerge in this evolving field. Three features of the networked social structure seem to deserve special attention in future research: network density, network centrality and the coevolution of network and behaviour. We briefly discuss some of the insights coming from existing studies and the potential role of network economics in advancing our understanding of these issues. In fact, while the existing research has provided convincing arguments for the importance of explicitly considering social networks in the study of natural resource management, a rigorous and careful analysis of the various trade-offs involved is still missing. The "economics" approach, based on the construction of fully fledged behavioural models together with explicit notions of equilibrium should prove useful in providing clear and the- 
oretically founded predictions of the role of the network topology for cooperative behaviour, and of the evolution of the network itself.

Network Density The hypothesis that a more connected society would imply greater potential for collective action is supported by many studies in the natural resource governance literature. Apart from the obvious observation that in order to cooperate on some issue some type of social contact is needed, the general idea is that more connections should enhance collaboration, ease monitoring and punishment, and help actors avoid conflicts and develop effective management strategies (see, for instance, Olsson et al. 2004). In the context of rural Kenya, King (2000) showed that fishermen communities characterised by a higher number of interactions among themselves and with government officials were relatively better able to deal with a series of unfavourable developments related to the fishery. Similar results emerge from Conley and Udry (2001)'s analysis of agricultural practices in Ghana, where high network density is associated with the development of new technologies and the diffusion of more sustainable management practices. However, careful inspection of the processes that govern behaviour on the network suggests that the intuitive but qualitative insight above may fail. For instance, as shown in Currarini (2007) (see also Sect. 3.3 below), by increasing the cohesiveness of the group, more connections may increase the outside option of potential free-riders, undermining the possibilities of cooperation. Moreover, large density may hinder co-operation by diffusing at a larger scale the information about the benefits of free riding, preventing therefore the creation of co-operative clusters. This was shown in the context of a model of evolutionary dynamics with myopic agents by Hanaki et al. (2007). Also, Bodin and Norberg (2005) have shown how large density in a regular network may induce large correlation in the strategy of myopic emulative agents, thereby increasing the odds of phase transitions in which the natural resource is overexploited and possibly exhausted.

Network Cohesiveness and Clustering Higher density may often come along with more cohesiveness and clustering. As we explained in Sect. 2, a cohesive network is one whose connectedness is little vulnerable to departures of agents or coalitions of agents. In particular, more cohesiveness means that it takes the departure of large coalitions to break up the network. So defined, cohesiveness guarantees high free-riding incentives to small defecting groups from a cooperating coalitions, who face large outside options due to the presence of positive spillovers (see, again, Sect. 3.3). In this case, cohesivenss is detrimental to cooperation. By the same spoken, cohesiveness lowers the incentives to defect when spillovers are negative, and facing a united residual coalition is detrimental to defectors' payoffs. It has also been shown by Hanaki et al. (2007) that high clustering can hinder cooperation by limiting the possibility that agents observe uncorrelated information. Since in their model agents learn and imitate their neighbours, it is best to observe uncorrelated information, since correlated information will often be biased in favour of the dominating strategy of "not cooperating" in prisoner's dilemma situations. This also implies that large networks, where the structure tends to be sparse both locally and globally, are more favourable to cooperation than small ones - another counter-intuitive conclusion. In order to better understand the role of clustering and cohesiveness, one should refer to more complex models of learning, in which both the imitation and the information acquisition functions of relational ties are at work. For instance, the promoting role of assortative matching for cooperation (see Watts 1999, 2002) should be contrasted with its averse effect on the speed of diffusion of information (see Golub and Jackson 2012), possibly resulting in the localization of cooperation and innovation in small and very cohesive groups.

Centrality While the notions of density and cohesiveness refer to the overall topology of social ties, and affect - as we have seen - the overall evolution of cooperation, centrality refers to the role of individual actors in the network. For example, by occupying certain 
central positions, some actors may be able to critically influence other relevant stakeholders, thus favouring (or blocking) the development of sustainable management initiatives. Stein et al. (2011) use the notions of degree centrality and betweenness centrality (see Sect. 2.1) to identify key players in the complex social and institutional landscape underpinning water governance in the Mkindo catchment, Tanzania. Within this context, the network of actors that either directly or indirectly influence water flows is a diverse set of players, ranging from local resource users and village leaders to higher-level governmental agencies, universities and NGOs. Results show that village leaders play a brokerage role in the network connecting water and land related activities within their respective village and, to some extent, across villages. The organisations with a formal mandate for the management of water resources, on the other hand, link across larger segments of the catchment, but are not well connected to local communities.

In general, identifying the most influential agents in the process of natural resource management may be more challenging than one would expect, both empirically and theoretically. Recently, Bonacich centrality has been shown to predict behaviour in the context of peer effects (Ballester et al. 2006), but behaviour may instead prevail at the periphery of the network when local negative externalities are present, as shown by Currarini et al. 2014. Empirically, this has been recorded, for instance, for the case of smoking intensity, which has been shown by Christakis and Fowler (2002) to be largest for agents far away from the core of the network. Interestingly, Ballester et al. (2006) have shown that network based policies aimed at reducing socially undesirable behaviour should target key-players who need not be central in the network. Other variants of centrality have been recently shown by Banerjee et al. (2013) to be relevant to identify which agents in a network would, if informed about a financial innovation, have the highest impact on overall adoption in society. Their study provides important insights on the differences between adoption and mere endorsement, and how these are related to the probability of diffusion. A similar analysis could be profitably applied to study the process of adoption of new technologies or behaviours in the context of natural resource management. This type of analysis would suggest efficient ways to integrate village leaders into formal water governance systems, and qualify the benefits of strengthening vertical links between local communities and governmental bodies operating at the district level.

Network Dynamics and the Coevolution of Behaviour As mentioned in Sects. 3.1.2 and 3.1.3, the existing studies on the role of networks on cooperative behaviour mostly focus on the evolution of behaviour on a fixed network structure, whose ties govern agents' interaction. A few recent papers suggest that the explicit consideration of the co-evolution of behaviour and of the underlying patterns of interaction may substantially enrich the steady state prediction of cooperation. Hanaki et al. (2007) study a dynamic system where agents' behaviour evolves by means of myopic and local imitation of other agents' performances, and the network evolves by means of myopic revisions of existing links. The evolution of social ties is such that co-operators will sever links with defectors, resulting in co-operators having more interactions and higher aggregate payoff than defectors. This would sustain cooperation in the long run, provided the average clustering of the network is low enough for defectors to remain isolated after a link is severed. The key insight here is that networks where social relations are more intertwined and clustered may discourage cooperation in the long run, precisely because defectors' behaviour, which is more profitable if conducted within a connected group, can be discontinued and isolated by link severance. As a result, groups of co-operators may survive and even attract isolated defectors, resulting in the enlargement of the scale of cooperation. In a related paper, Skyrms and Pemantle (2000) study the evolution of repeated behavior within an evolving network, showing that the explicit modeling of the 
network dynamics may qualitatively change the behavioral prediction in steady state. By only looking at random dyadic relations, and neglecting the role of indirect relation, this paper neglects important features of networked interaction, but clearly highlights the importance of co-evolution of behavior and social structure.

\subsection{Networks, Coalitions and International Agreements}

In this section we discuss two issues in international cooperation that would benefit from the explicit consideration of networks and from the application of notions developed in network economics. In a nutshell, in Sect. 3.4.1 we discuss new insights on the stability of cooperation that would obtain from the explicit consideration of countries' bilateral relations within a cooperating coalition (here represented by a network). The focus here is on the way in which the structure of bilateral relations within a cooperating coalition affects its stability. In Sect. 3.4.2 we consider the process of coalition-building through sequential bilateral contacts, and discuss the trade-offs between centralization and delegation of these contacts. The focus here is, therefore, on the formation of a cooperating coalition and on the process that leads to it.

\subsubsection{The Internal Structure of Environmental Coalitions}

A common, yet restrictive assumption in the economic literature of IEAs is that countries are symmetric. When taken into consideration, asymmetries are typically modelled as differences in terms of costs and benefits of emission abatement. However, due to their history of political, economic and cultural interactions, countries may also differ with respect to their relationship and role within the process of building up cooperation (see Sect. 3.4.2). Because of these differences, even within a cooperating coalition, certain countries may find it easy to communicate and agree on proposals, while other countries may have little, if any, relations. These differences in bilateral relations within the coalition are likely to characterize countries' relations should the coalition break down and, with it, their possibilities of cooperation after the break up. If, for instance, two countries A and B manage to cooperate within a larger coalition only thanks to the mediation of a third country $\mathrm{C}$, these two countries would probably find it difficult to cooperate if $\mathrm{C}$ were to leave the coalition.

To put things more formally, we associate with the environmental coalition $S$ a set of (possibly weighted) bilateral links, expressing, for each pair of countries in $S$, the strength of their diplomatic, political and economic relationship. In the simplest case, we may think of a $\{0,1\}$ undirected network, where countries either communicate or not within $S$. The cornerstone of our analysis is the mechanism described above: by describing countries' bilateral relations, the network predicts countries' cooperation possibilities in case the coalition should break apart. Consider, for instance, country 2 in the left panel of Fig. 8, mediating all other bilateral relations; 2's defection from the three-country coalition would cause a total breakdown of

Fig. 8 Internal structures of a 3-country coalition

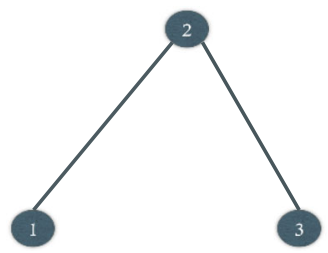

(a)

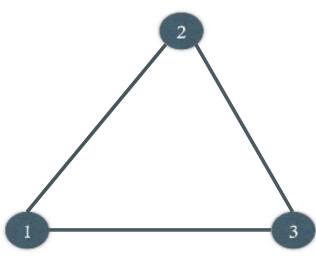

(b) 
cooperation, as countries 1 and 3 would not be able (or would face prohibitively high costs) to communicate. If 2 were to defect from a coalition internally structured as in the right panel of Fig. 8, a smaller cooperating coalition with 1 and 3 as members would be possible.

What matters for our argument is the observation that, in the presence of spillovers, what a country expects to obtain by defecting from a coalition crucially depends on the expected patterns of cooperation after the defection. Free riding incentives are clearly maximal when the remaining countries are expected to stay together and continue to cooperate. This is the so-called "delta" assumption, and leads to the prediction of an endemic instability of cooperation when spillovers are positive (as in the case of environmental agreements). Free riding incentives are, instead, minimized when other countries are expected to stop cooperating altogether after a defection - the "gamma" assumption - in which case global cooperation has been shown to be possible (see Chander and Tulkens 1997). By specifying the internal structure of a coalition, the network pins down countries' expectations on post-defection scenarios, and therefore their incentives. For instance, the gamma assumption is naturally associated with country 2's defection in network (a) of Fig. 8, while the delta assumption would appropriately describe expectations after 1's and 3's defections. In general, the defection of a sub-coalition $S \subset N$ from the set $N$ internally organized according to the connected network $g$ would be followed by the partition $\pi\left(\left.g\right|_{N \backslash S}\right)$ of the remaining players $N \backslash S$, where each element of the partition corresponds to a component of the sub-network $\left.g\right|_{N \backslash S}$ (see Sect. 2.1).

By shaping defectors' incentives, relational networks endow a cooperating coalition with specific stability properties that depend on the sign of coalitional spillovers. In Fig. 8, for instance, the "star" network of the left panel provides all players with lower incentive to defect under positive spillovers, and with higher incentives under negative spillovers, than the complete network on the right panel. More generally, adding links to a given network always has the effect of lowering incentives to defect under negative spillovers, and of increasing these incentives under positive spillovers. This suggests that minimally connected structures would endow the coalition with strong stability properties under positive spillovers.

Additionally, a very sparse internal structure would also limit the possibilities of coalitional members to coordinate on defections. In the star network of Fig. 8, for instance, countries 1 and 3 would not be able to coordinate on a joint defection, unless they get player 2 involved. This is not the case in the complete network, where 1 and 3 can autonomously take joint decisions. The effect of the network on coordination was analysed by Demange (2004) in standard cooperative games and by Currarini (2007) in games with spillovers. A basic insight is that, under positive spillovers, sparse networks maximize coalitional stability by both limiting the number and the profitability of potential defections. Things are more ambiguous under negative spillovers: while sparse networks limit the number of potential defections, they maximize their profitability, resulting in a trade-off that leaves space for intermediate structure with average density.

While the above discussion stresses the role of the relational network in shaping players' outside options, there are other ways in which the network is likely to affect players' bargaining power within the coalition. In the left panel of figure 8 , while under positive spillovers player 2 has a low outside option due to limited free riding possibilities, he is nevertheless responsible for keeping the coalition united and, therefore, for generating the gains from cooperation. This should increase its bargaining power compared to the complete network (right panel), where 2 is not in such a pivotal position. This is indeed recognized by various allocation rules that take account of the network, such as the Myerson Value, an extension of the Shapley Value to cases where players' cooperation possibilities are described by a network. The main insight here is that while pivotal players enjoy a stronger bargaining position 
under negative spillovers, a trade-off obtains under positive ones, where pivotal players, who play an essential role within the coalition, end up facing low outside options and, as a result, weaker bargaining power. More research is needed to fully understand the interplay of the different roles of the network in shaping agents bargaining power, and how this interplay affects the stability of environmental coalitions.

\subsubsection{Delegation and Centralization in the Build-up of Environmental Coalitions}

The process by which environmental coalitions are formed can be varied and multifaceted, and the timing and framing of negotiations is likely to matter for the final success of cooperation. In particular, large coalitions are likely to be built gradually, with a limited number of very committed members as first signatories, who then adopt various strategies to enlarge the coalition.

The gradual process through which coalitions are built, and the amount of strategy involved, are not the focus of the two main approaches to environmental coalitions, based on the notion of core of a cooperative game (see Chander and Tulkens 1997), or on simultaneous non cooperative models of coalition formation (as in Carraro and Siniscalco 1993 and Barrett 1994). In many instances of international environmental cooperation, however, one or more countries have played the role of perpetrators of the process, either because more inclined to solve global environmental problems, or because traditionally playing a leading role in the international arena. Such countries face the task of building up a larger coalition by means of several and successive individual contacts with other perspective members, through complex negotiation processes. The design of such bilateral contacts is a crucial element of cooperation, and attains to the timing of such contacts, their degree of centralization and delegation, the personal involvement and commitment of perpetrators and of perspective members. The perpetrator may, for instance, opt for multiple and simultaneous contacts with most of the other potential members, adopting therefore a centralized procedure of coalition building. Alternatively, it may identify a restricted set of players to contact in a first stage of negotiation, and delegate to these players the task of further enlarging the coalition.

Both centralization and delegation have plausible pros and cons. Advocates of centralization would probably stress the importance of a widespread use of the authority and charisma of the perpetrator, whose central role would be interpreted as signal of its commitment to the cooperation process. Delegation would probably be preferred when diplomatic, geographical and historical relations between countries are very heterogeneous, and the initial perpetrator would lack the necessary information and/or diplomatic strength to successfully negotiate with certain potential new members. In these cases, the perpetrator may better serve the final goal of global cooperation by delegating the creation of new contacts.

The choice between delegation and centralization involves other, less obvious, aspects that are strictly related to the economics of cooperation and to the resulting patterns of strategic interaction. In this section we discuss such aspects, frame them in a stylized example of coalitional externalities, and claim that a general analysis of these issues would greatly benefit from the use of network formation theory and from our knowledge of strategic interdependence in networks. To fix ideas, consider the following three-player example, developed in full detail in Currarini and Feri (2006). A perpetrator $i$ has the task of building up a coalition with two other players, $j$ and $k$. The benefits from cooperation are captured by a partition function $v$, mapping each partition of the set of players into a vector of payoffs, specifying an aggregate payoff for each coalition in that partition. Formally, we let $v(S, \pi)$ denote the value generated by $S$ in the partition $\pi$. In our example, we set $v(\{i\},\{i, j, k\})=v(\{j\},\{i, j, k\})=v(\{k\},\{i, j, k\})$ and $v(\{i j\},\{i j, k\})=v(\{i k\},\{i k, j\})=v(\{j k\},\{i, j k\})$ by symmetry. We also assume 
Fig. 9 Centralised (left) versus decentralised (right) contacts

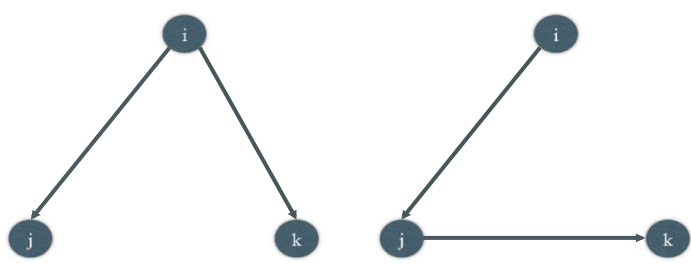

that the grand coalition $\{i j k\}$ is efficient, by this meaning that it generates a higher aggregate payoff than any other partition of the players' set: $v(\{123\},\{123\}) \geq \sum_{S \in \pi} v(S, \pi), \forall \pi$.

The perpetrator $i$ designs the structure of his contacts with $j$ and $k$. Either $i$ contacts $j$ and $k$ simultaneously, proposing to form a coalition of three players, or sequentially, contacting $j$ first, proposing him to join the forming coalition, and delegating him the task of enlarging the coalition to $k$. In other words, $i$ admits $j$ in the coalition, and transfers to $j$ the technology to negotiate with $k$. The assumption that the perpetrator can commit not to contact agent $k$ when delegating to agent $j$ the contracting power is crucial and considerably simplifies the equilibrium analysis and allows us to get a first very sharp intuition. The two scenarios are illustrated in Fig. 9.

In the first centralized scenario, $j$ and $k$ simultaneously receive an offer. For both of them to accept, the offered monetary payoff has to exceed the outside option given that the other has accepted. These outside options are $v(\{j\},\{j, i k\}=v(\{k\},\{k, i j\}$. If the perpetrator delegates, then player $j$ needs to receive at least what he would get by rejecting the offer, which is $v(\{j\},\{i, j, k\})$. Player $k$ 's payoff when contacted by $j$ would instead be at least $v(\{k\},\{i j, k\}$, his outside option if rejecting to join the coalition.

Summing up, the perpetrator needs to give up different slices of the total cake in the two alternative regimes: by centralizing contacts, $i$ gives up $v(\{j\},\{j, i k\}+v(\{k\},\{k, i j\}$; by delegating, $i$ gives up $v(\{j\},\{j, i, k\})+v(\{k\},\{k, i j\}$. Which regime is preferred by the perpetrator clearly depends on whether $v(\{j\},\{j, i, k\}>v(\{j\},\{j, i k\}$ or, instead, $v(\{j\},\{j, i, k\}<v(\{j\},\{j, i k\}$. In the terminology of coalitional games, it depends on whether agents face negative or positive coalitional spillovers. In particular, the perpetrator will prefer centralized contacts when spillovers are negative, and sequential contacts when spillovers are positive. Also, when there are intrinsic reasons to centralize contacts (based, as we said, on the perpetrator authority), there is a trade-off between these reasons and the strategic incentives to free ride in a centralized process, and this trade-off may be resolved in favour of delegation the stronger free riding incentives and/or the weaker the perpetrator's authority.

The role of externalities on outside options, bargaining power and the resulting structure of contracts has been stressed in various papers in the contracts literature. Genicot and Ray (2006) suggest that the presence of negative externalities may induce the principal to first contract a subset of players, and then extend contracts to other individual players - a sort of divide and conquer strategy. Similar insights are present in Galasso (2008). The main insight here is that the first set of contracts has the purpose of decreasing the outside options of the remaining players, who are then contracted at better conditions for the principal. The reason why a fully centralised structure is not optimal in these papers is that players can coordinate before responding to the principal's offer. Another difference with our stylized example above is that the principal cannot commit to transferring the contracting power to the agents. A similar mechanism underlies the analysis of centralized contracts with externalities by Segal (1999), where is shown that positive externalities may induce the principal to delegate inefficiently low activity levels, in the attempt to lower agents' outside options and retain 
a larger share of the social surplus. A general setting that extends the three-player example described above to many agents is the sequential "link formation and bargaining" game in Currarini and Morelli (2000). There, the sequential formation of links has been shown to induce efficiency in the absence of externalities, thus overlooking the free riding incentives and their effects on coalition formation. Also, although in that paper the principal can decide whether to simultaneously offer a link to all agents or to delegate to the second agent in the protocol the task to form further links, the principal cannot retain the exclusive right to propose contract. The centralized contracting situation realizes therefore only if all agents reciprocate the principal's link offer and do not form links among themselves.

\section{Conclusions}

In this paper we have discussed how network economics can help to model and analyse a variety of environmental problems, and what new insights can result. In this final section, we wish to offer some further thoughts on the relationship between network density and cooperative behaviour; an issue, which arises, in one way or another, in all the applications we have considered in this survey. Specifically: what type of local interaction promotes cooperative (and socially efficient) behaviour? Are dense networks more conducive to cooperation than sparse one? Is symmetry in social relations beneficial to pro-social behaviour? In Sect. 3.1, we have seen how the use of evolutionary game theoretic models has lead to the conclusion that cooperation is harder to sustain in large populations. This is due to the large benefits from defection when the number of interactions increases. Hence, this is not a statement about the size of overall population, but rather one about the number of social relations. Increasing population size with a constant number of interactions (that is, decreasing network density), does not affect the defectors' incentives; however, as pointed out by Coleman (1988), the degree of clustering (or closure) in social relations matters for cooperation, as mutual acquaintances provide reinforcement and monitoring of virtuous behaviour. Jackson and Yariv 2011 reach a similar conclusion in a fully fledged game theoretical model of favor exchange, which shows that forward looking rational agents cooperate only if they share one or more common neighbors. So, a larger total population may hinder cooperation if it comes at the cost of lower clustering. This common view has been challenged by works, such as Hanaki et al. (2007), where sparseness is claimed to promote cooperation by favoring the enlargement of cooperative groups to defectors. Here the main force promoting large-scale cooperation is the asymmetry of social relations, so that isolated defectors may turn into co-operators by imitating well-connected (and happier) co-operators. Arguments in favour of asymmetries in the network structure also come from other recent papers, where scale free distributions are found to favour pro-social behaviour (see Sect. 3.1.2). In Sect. 3.4 we have then discussed a different mechanism relating the density of a network of cooperators with the stability of cooperation. We have there studied a construct where the components of a network represent groups of cooperators (the "coalitions" of cooperative game theory), endowed with an internal structure of bilateral relations. We have argued that the topology of a component affects the incentives of its members to break out by shaping their outside options. This happens when defectors use the network topology to predict future cooperative scenarios after their defection. Dense networks, robust to the deletion of nodes, favor defectors in public good games, where the disruption of cooperation would act as a punishment, and are therefore little apt to sustain cooperation. A similar insight is present in Hanaki et al. (2007), where high clustering prevents cooperation by limiting the possibility of indi- 
vidual agents to exclude defectors from the benefits of interacting with cooperators. By the same token, when the disruption of cooperation is beneficial for defectors, as in pure conflict or bargaining games, dense network structures promote cooperation by ensuring that some cohesive structure will endure after the defection of one or more players. In sum, the nature of the relationship between network density and cooperation ultimately depends on the type of interaction at work: high density tends to favor cooperative behavior when spillovers are negative across groups (the typical case in environmental games), and to hamper it when spillovers are positive.

Local interaction and network structures seem to bear potential applications in other environmental problems that we have not covered here, including multi-issues environmental negotiations, issue linkage, trans-boundary pollution problems, biodiversity and conservation, peer effects in health related behaviour with externalities (such as smoking), fisheries management, risk assessment and others. We hope that the present paper can stimulate research on these topics, both theoretical and applied, explicitly embedding networks in the traditional models of environmental economics. Some of these applications are the subject of our current research.

\section{References}

Acheson JM (2003) Capturing the commons: devising institutions to manage the maine lobster industry. University Press of New England, Lebanon

Alonso-Sanz R (2009) Memory versus spatial disorder in support of cooperation. BioSystems 97:90-102

Axelrod RM (1984) The evolution of cooperation. Basic Books, New York

Baland J-M, Bardhan P, Bowles S (eds) (2006) Inequality, cooperation, and environmental sustainability. Princeton University Press, Princeton

Baland J-M, Platteau J-P (1996) Halting degradation of natural resources. Is there a role for rural communities?. Clarendon Press, Wotton-under-Edge

Ballester C, Calvó-Armengol A, Zenou Y (2006) Who's who in networks. wanted: the key player. Econometrica 74(5):1403-1417

Banerjee A, Chandrasekhar AG, Duflo E, Jackson MO (2013) The diffusion of microfinance. Science 341(6144)

Barabasi A (1999) Emergence of scaling in random networks. Science 286(5439):509-512

Barberis N, Shleifer A (2003) Style investing. J Financ Econ 68:161-199

Bardhan P, Ghatak M, Karaivanov A (2006) Wealth inequality and collective action. J public econ 91(9):18431874

Barrett S (1994) Self-enforcing international environmental agreements. Oxf Econ Paper 46:878-894

Bass FM (1969) A new product growth for model consumer durables. Manag Sci 15(5):215-227

Bloch F, Jackson MO (2007) The formation of networks with transfers among players. j econ theory 133(1):83110

Bodin A, Norberg J (2005) Information network topologies for enhanced local adaptive management. Environ Manage 35(2):175-193

Calvo-Armengol A (2004) Job contact networks. J Econ Theory 115:191-206

Calvo-Armengol A, Jackson MO (2004) The effects of social networks on employment and inequality. Am Econ Rev 94(3):426-454

Carraro C, Siniscalco D (1993) Strategies for the international protection of the environment. J Public Econ 52(3):309-328

Chander P, Tulkens H (1997) The core of an economy with multilateral environmental externalities. Int J Game Theory 26(3):379-401

Chen X-J, Fu F, Wang L (2008) Interaction stochasticity supports cooperation in spatial prisoner's dilemma. Phys Rev E. doi:10.1103/physreve.78.051120

Christakis N, Fowler JH (2002) The collective dynamics of smoking in a large social network. N Engl J Med 358(21):2249-2258

Chung NN, Chew LY, Lai CH (2013) Influence of network structure on cooperative dynamics in coupled socio-ecological systems. Europhys Lett 104(2):28003-28009 
Coleman JS (1988) Free riders and zealots: the role of social networks. Sociol Theory 6:52-57

Conley T, Udry C (2001) Social learning through networks: the adoption of new agricultural technologies in Ghana. Am J Agric Econ 83:668-673

Currarini S (2007) Network design in games with spillovers. Rev Econ Design 10(4):305-326

Currarini S, Jackson MO, Pin P (2009) An economic model of friendship: homophily, minorities and segregation. Econometrica 77(4):1003-1045

Currarini S, Jackson MO, Pin P (2010) Identifying the roles of race-based choice and chance in high school friendship network formation. Proc Nat Acad Sci 107(11):4857-4861

Currarini S, Feri F (2006) Centralization versus delegation: the role of externalities. Res Econ 60(2):112-119

Currarini S, Fumagalli E, Panebianco F (2014) Games on Networks: Direct Complements and Indirect Substitutes. Discussion Papers in Economics 13/04, Department of Economics, University of Leicester

Currarini S, Morelli M (2000) Network formation with sequential demands. Rev Econ Des 5 (3)

Demange G (2004) On group stability in hierarchies and networks. J Polit Econ 112(4):754-778

Dixit A (2003) Clubs with entrapment. Am Econ Rev 93(5):1824-1829

Dutta B, Mutuswami S (1997) Stable networks. J Econ Theory 76(2):322-344

Ebel H, Bornholdt S (2002a) Coevolutionary games on networks. Phys Rev E. doi:10.1103/PhysRevE.66. 056118

Ebel H, Bornholdt S (2002b) Evolutionary games and the emergence of complex networks ( arXiv:cond-mat/0211666)

Elliott M, Golub B, Jackson M (2013) Financial networks and contagion. Review 104(10):3115-3153

Galasso A (2008) Coordination and bargaining power in contracting with externalities. J Econ Theory 143: $558-570$

Galeotti A, Goyal S, Jackson MO, Vega-Redondo F, Yariv L (2010) Network games. Rev Econ Stud 77(1): $218-244$

Genicot G, Ray D (2006) Contracts and externalities: how things fall apart. J Econ Theory 131:71-100

Gilles JL, Hammoudi A, Mahdi M (1992) Oukaimedene, morocco: a high mountain agdal. In: Bromley D et al (eds) Making the commons work: theory, practice, and policy. ICS Press, Washington, pp 229-246

Gladwell M (2000) The Tipping point: how little things make a big difference. Little Brown, New York

Golub B, Jackson MO (2012) How homophily affects the speed of learning and best-response dynamics. Q J Econ 127(3):1287-1338

Goyal S, Moraga-Gonzalez JL (2001) R\&D networks. RAND J Econ 32(4):686-707

Granovetter M (1978) Threshold models of collective behavior. Am J Sociol 83(6):1420-1443

Gräser O, Xu C, Hui PM (2009) Disconnected-connected network transitions and phase separation driven by co-evolving dynamics. EPL 87:38003

Griliches Z (1957) hybrid corn: an exploration in the economics of technological change. Econometrica 25(4):501-522

Hanaki N, Peterhansl A, Dodds PS, Watts DJ (2007) Cooperation in evolving networks. Manage Sci 53(7):1036-1050

Hardin G (1968) The tragedy of the commons. Science 162(3859):1243-1248

Hauert Ch, Doebeli M (2004) Spatial structure often inhibits the evolution of cooperation in the Snowdrift game. Nature 428:643-646

Heal G, Kunreuther H (2012) Tipping Climate Negotiations. In: Climate Change and Common Sense: Essays in Honour of Tom Schelling, edited by Robert W. Hahn and Alastair Ulph. Joseph E. Aldy

Hofbauer J, Sigmund K (1998) Evolutionary games and population dynamics. Cambridge University Press, Cambridge

Hubert F, Cobanli O (2012) Pipeline Power. mimeo

Ilkiliç R (2011) Networks of common property resources. Econ Theor 47:105-134

Jackson MO, Yariv L (2011) Diffusion, strategic interaction, and social structure. In Benhabib, Bisin, Jackson (eds) The handbook of social economics, North Holland Press, Amsterdam

Jackson MO (2008) Social and economic network. Princeton University Press, Princeton

Jackson MO, van den Nouweland A (2005) Strongly stable networks. Games Econ Behav 51(2):420-444

Jackson MO, Wolinsky A (1996) A strategic model of social and economic networks. J Econ Theory 71(1):4474

King A (2000) Managing Without Institutions: The Role of Communication Networks in Governing Resource Access and Control, Ph.D. thesis, University of Warwick

Kranton RE, Minehart DF (2001) A theory of buyer-seller networks. Am Econ Rev 91(3):485-508

Lade SJ, Tavoni A, Levin SA, Schlüter M (2013) Regime shifts in a social-ecological system. Theor Ecol $6(3): 359-372$

Leibenstein H (1950) Bandwagon, snob, and veblen effects in the theory of consumers' demand. Quart J Econ 64(2):183-207 
Marchiori C, Sayre Stratton S, Simon L (2012) Bargaining and devolution in the upper guadiana basin. Environ Resour Econ 51(3):453-470

McKean MA (1986) Management of Traditional Common Lands in Japan. In: Proceedings of the Conference on Common Property Resource Management, ed. National Research Council, pp. 533-90, National Academies Press

McKean MA (2000) Common property: what is it, what is it good for, and what makes it work? In: Gibson CC, McKean MA, Ostrom E (eds) People and forests: communities, institutions, and governance. MIT Press, Boston, pp 27-50

Molle F, Wester P, Hirsch P, Jensen JR, Murray-Rust H, Paranjpye V, Pollard S, van der Zaag P (2007) River basin development and management. In: Molden D (ed) Water for food, water for life: a comprehensive assessment of water management in agriculture. Earthscan, Routledge

Myerson RB (1977) Graphs and cooperation in games. Math Operat Res 2:225-229

Netting RMC (1981) Balancing on an alp: ecological change and continuity in a swiss mountain community. Cambridge University Press, Cambridge

Nowak MA, Sasaki A, Taylor C, Fudenberg D (2004) Emergence of cooperation and evolutionary stability in finite populations. Nature 428:646-650

Nowak MA, May RM (1992) Evolutionary games and spatial chaos. Nature 359:826-829

Nugent JB, Sanchez N (1999) The local variability of rainfall and tribal institutions: the case of Sudan. J Econ Behav Organ 39:263-291

Ohtsuki H, Hauert C, Lieberman E, Nowak MA (2006) A simple rule for the evolution of cooperation on graphs. Nature 441(7092):502-505

Olson M (1965) The logic of collective action: public goods and the theory of groups. Harvard University Press, Cambridge

Olsson P, Folke C, Berkes F (2004) Adaptive comanagement for building resilience in social and ecological systems. Environ Manage 34:75-90

Olsson P, Folke C, Hughes TP (2008) Navigating the transition to ecosystem-based management of the Great Barrier Reef, Australia. Proc Nat Acad Sci 105:9489-9494

Ostrom E, Burger J, Field CB, Norgaard RB, Policansky D (1999) Revisiting the commons: local lessons, global challenges. Science 284(5412):278-282

Ostrom E (2003) How types of goods and property rights jointly affect collective action. J Theor Polit 15(3):239-270

Platteau J-P (1991) Traditional systems of social security and hunger insurance: past achievements and modern challenges. In: Ahmad E et al (eds) Social security in developing countries. Clarendon Press, Wottonunder-Edge, pp 112-170

Pretty J, Ward H (2001) Social capital and the environment. World Dev 29:209-227

Rand DG, Nowak MA, Fowler JH, Christakis NA (2014) Static network structure can stabilize human cooperation. Proc Nat Acad Sci 111(48):17093-17098

Rogers EM (1995) Diffusion of innovations. Simon \& Schuster International, New York

Ryan B, Gross N (1943) The diffusion of hybrid seed corn in two iowa communities. Rural Sociol 8(15):15-24

Santos FC, Pacheco JM (2005) Scale-free networks provide a unifying framework for the emergence of cooperation. Phys Rev Lett. doi:10.1103/PhysRevLett.95.098104

Santos FC, Pacheco JM, Lenaerts T (2006) Evolutionary dynamics of social dilemmas in structured heterogeneous populations. Proc Nat Acad Sci 103:3490-3494

Santos FC, Pinheiro FL, Lenearts T, Pacheco JM (2012) The role of diversity in the evolution of cooperation. J Theor Biol 299:88-96

Schoonmaker Freudnberger M (1993) Regenerating the Gum arabic economy: local-level resource management in northern Senegal. In: Friedmann J, Rangan H (eds) In defence of livelihood: cooperative studies on environmental action. Kumarian Press, Boulder, pp 52-78

Scott J (2000) Social network analysis: a handbook, 2nd edn. Sage, Newberry Park

Segal Y (1999) Contracting with externalities. Q J Econ 114(2):337-388 (May, 1999)

Singleton S (1999) Common problems, collective action and efficiency: past and present institutions of governance in pacific northwest salmon fisheries. J Theor Polit 11(3):367-391

Skyrms B, Pemantle R (2000) A dynamic model of social network formation. Proc Nat Acad Sci 97(16):93409346

Stein C, Ernstson H, Barron J (2011) A social network approach to analyzing water governance: the case of the mkindo catchment, Tanzania. Phys Chem Earth 36:1085-1092

Szolnoki A, Perc M (2009c) Resolving social dilemmas on evolving random networks. doi:10.1209/ $0295-5075 / 86 / 30007$

Tavoni A, Schlüter M, Levin SA (2012) The survival of the conformist: social pressure and renewable resource management. J Theor Biol 299(21):152-161 
Trawick P (2003) Against the privatization of water: an indigenous model for improving existing laws and successfully governing the commons. World Dev 31(6):977-996

Tulkens H, Chander P (1997) The core of an economy with multilateral environmental externalities. Int J Game Theory 26(3):379-401

Watts DJ (1999) Small worlds: the dynamics of networks between order and randomness. Princeton University Press, Princeton

Watts DJ (2002) A simple model of global cascades on random networks. Proc Nat Acad Sci 99(9):5766-5720

Weir S, Knight J (2004) Externality effects of education: dynamics of the adoption and diffusion of an innovation in rural ethiopia. Econ Dev Cult Change 53(1):93-113

Young HP (2009) Innovation diffusion in heterogeneous populations: contagion. Soci InfluSoc Learn Am Econ Rev 99:1899-1924 